\title{
Arthroscopic suture fixation of comminuted tibial spine fractures-a prospective study
}

\author{
Kumar $S^{1}$, Kushwaha S.S ${ }^{2}$, Singh $S^{3}$, Kumar $D^{4}$, Sharma $V^{5}$ \\ ${ }^{1}$ Dr Kumar Shantanu, Assistant Professor, ${ }^{2}$ Dr Sudhir Shyam Kushwaha, Senior Resident, ${ }^{3}$ Dr Shailendra Singh, \\ Assistant Professor, ${ }^{4}$ Dr Deepak kumar, Assistant Professor, ${ }^{5}$ Dr Vinnet Sharma, Professor \& Head. All are affiliated \\ with Department of Orthopaedics, King George's Medical University, Lucknow, UP, India.
}

Address for correspondence: Dr Sudhir Shyam Kushwaha, Email: sudhirshyamkushwaha@gmail.com

\begin{abstract}
Introduction: Tibial spine fractures are common injuries and various techniques have been described for the fixation. The purpose of the article is to make familiar with thearthroscopic fixation of the anterior cruciate ligament bony avulsion injuries using non absorbable sutures and simple methods and instruments. Adequate reduction, anterior cruciate ligament integrity and regaining the bio-mechanics of the knee are the ultimate goal to be achieved failing which non union and malunion ensues. This technique has resulted in achieving excellent results and thus avoiding complications like joint laxity, flexion deformity \& pain. Methods: Twenty one patients underwent arthroscopic suture fixation of the comminutedtibial spine fractures. Mean age of the patient was 24.28 years (range 16 to 38 years). All of the patients belonged to the Meyer type IV. The mean duration of follow up was 15.5 months with a minimum of one year follow-up. Results: All patients showed negative Lachman test except one having grade 1 positive lachman. No patient showed extension loss. $90.4 \%(n=19)$ patients had an IKDC grade A while two patients belonged to grade B. All patients returned to their previous activity level. Conclusion: The above excellent results show that arthroscopic suture fixation of the comminutedtibial spine is promising and reliable technique to achieve the best biomechanics of the knee.
\end{abstract}

Keywords: Tibialspine, Arthoscopic suture fixation, ACL

\section{Introduction}

Tibial spine fractures or anterior cruciate ligament bony avulsion injuries are common injuries which result generally from falls, road traffic accidents and footballinjuries [1]. The first documentation can be traced back to 1875 . The mechanism of the injury is hyperextension of the knee [2] and majority of the patients fall in the children and adolescentsage group although well described in adults too [3]. This can be explained on the basis that bone gives way before the ligaments and hence common in children.

The most widely used classification to describe tibial spine fractures is given by Meyers and McKeever's which isa radiologic classification system [4]. Type I fracture is an undisplaced fracture of tibialeminence, type II is partially displaced with intact posterior hinge, andType III fracture is completely displaced fracture

Manuscript received $16^{\text {th }}$ May 2016

Reviewed: $27^{\text {th }}$ May 2016

Author Corrected: $14^{\text {th }}$ June 2016

Accepted for Publication $27^{\text {th }}$ June 2016 and there is no contact of avulsed fragment to the bone bed and reduction in these types of injuries is difficult because of the transverse meniscal ligament preventing the fragment to fall back. Zaricznyj proposed a fourth category (type IV) for comminuted avulsed fragment [5]. According to Rockwood et al type I and II can be managed conservatively [6]. Kocher and co-worker [7] found $65 \%$ and Senekovic and Veselko [8] found $100 \%$ of type III fractures had intermeniscal ligament, anterior horn of medial meniscus, or anterior horn of the lateral meniscus incarcerated within the fracture and thus lower likelihood of the success of the close reduction. So, looking onto the difficulties,most authors have suggested operative fixation of type III and type IV.

The various options available for fixation of the tibial spine areopen reduction with casting, open reduction with internal fixation, arthroscopic reduction with casting, and arthroscopic reduction and internal fixation 
with sutures, cancellous screws, stainless steel wires, Kirschner wires, and anchor sutures.

Overall the aim is to achieve the restoration of biomechanics and the joint congruity and cruciate ligament integrity failing which may result in non-union or malunion leading to complications like joint instability, flexion deformity [9]. Arthroscopictreatment is the gold standard nowadays. Recent trends show a shift towards less use of the metal hardwares which need second surgery for the removal and thus hopping to the use of various absorbable and non-absorbable sutures which has also got the advantages of minimal to none physeal damage in children.

In our study we present a simple technique of arthroscopic fixation of comminutedtibial spine fractures (type IV) using non absorbable ultabraidsutures.

\section{Material and Methods}

The study was conducted at department of orthopaedics, KGMU, Lucknow. In our study we includedtwenty one patients with isolated Meyer type IV comminuted fractures of the tibialspinepresenting to ouroutpatient departmentall these patients had injuryofless than 7 dayspatients with injury duration more than 7 days were excluded from the study. Patients with other associated injuries of the lower limb, patients with medical comorbiditied were excluded from the study. Patients were followed at 6 weeks, 12 weeks, 6 month and 1 year. Subjective evaluation of knee function was done by IKDC scoring and objective evaluation of knee function was done by Lachman test. Radiological evaluation was done by $\mathrm{x}$ ray at each follwoup to look for union. statistical analysis was done to get the results of the study.

Surgical Technique: Under regional anaesthesia and using the pneumatic tourniquet, the standard anterolateral portal is made and joint is lavaged clearing the haematoma and debris until clear vision is established. Arthroscopic inspection of the knee joint is done and other injuries such as meniscal and chondral lesions are ruled out. Probing is done to assess the avulsed fragment regarding size which often appears to be larger as shown in radiographs due to the cartilage component. Usually the transverse inter-meniscal ligament is found to be entrapped between the tibial spine fragment and the crater which needs to be brought back in its original placeas it hinders the reduction using either probe or traction suture. Using shaver and radio-frequency wand fracture site is cleared of haematoma whereas debris and fat pad was shaved. Fracture crater is cleaned and additional curettage of cancellous bone is done by using burr to achieve bleeding surface and therefore achieving appropriate reduction. Now the reduction of the fragment is doneusing the tibial ACL zig passed from the antero-medial portal and under arthroscopic guidance now tibial tunnel is drilled medial to thetibial tuberosity using the $2 \mathrm{~mm} \mathrm{~K}$-wire while ACL zig is placed in the medial-most edge of the avulsion crater. Precaution is taken here to place the guide at steep angle (usually 55-60 degrees) so that the K-wire comes through the medial part of the avulsed bone and the ligament for easier retrieval of sutures later on. Once the tip of the K-wire is visible it is left in situ and now the ACL zig is placed on the lateral most edge of the avulsion crater and another $\mathrm{K}$ wire is drilled in the similar fashion and is left in situ.

Now a looped prolene suture (no3) is passed into a lumbar puncture (LP) needle, K-wires are withdrawnand the LP needle loaded with the proleneis pushed through the tibial tunnels and once the LP needle tips are visualised, theprolene sutures are pushed inside more through the lumbar puncture needle so that the loop opens up (Fig 1,2). Further by using an epidural needle loaded with ultrabraidsututres is inserted percutaneously in the joint from medial sideabove the medial meniscusand is advanced so that needle tip passes through the medial loop. Now the loaded epidural cannula is advanced so that it pierces the anterior cruciate ligament substance as close to fragment as possible and as posteriorly as possible is till it comes outpostero-laterally from the ACL, when the epidural cannula tip is seen, the ultrabraidsuture is pushedmore. The grasper is passed through the antero-medial portal and is progressed through the lateral loop of the proleneloop and the tip of the ultrabraidsuture passed from the ACL substance is pulled using the grasper and is taken outside the anteromedial portal. The whole step is repeated again but this time the anterior substance of the ACL is pierced. The two prolenesutures are shuttled by the non-absorbable ultrabraid sutures (Fig 3). Now the needles from the tibial tunnel were removed and the prolene loops are pulled out which along with them brings the Ultrabraidsutures out of the joint. And now the ultrabraid sutures are pulled tightly which reduces the tibial spine fracture segment back into the place and integrity of the ACL is maintained again (Fig 4). In full extension, roof impingement is checked. Now the ends of the ultabraid sutures are knotted over the tibial bony bridges between the tunnels created by the K-wires. Once again the knee joint is inspected and tension of the ACL is confirmed 


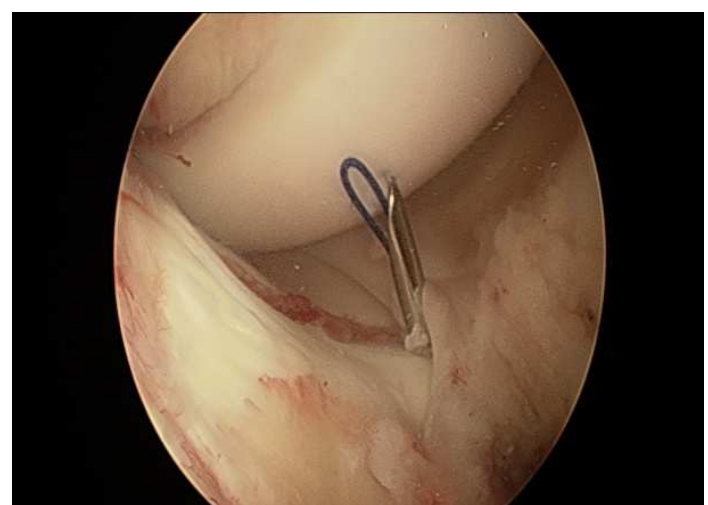

Fig 1: PDS suture passed through LP needle

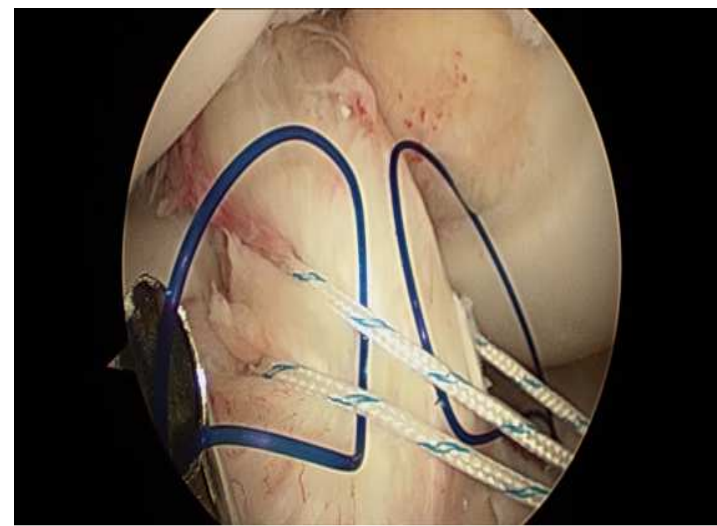

Fig 3:Ultrabraid sutures shuttled through both PDS sutures and taken out for tying

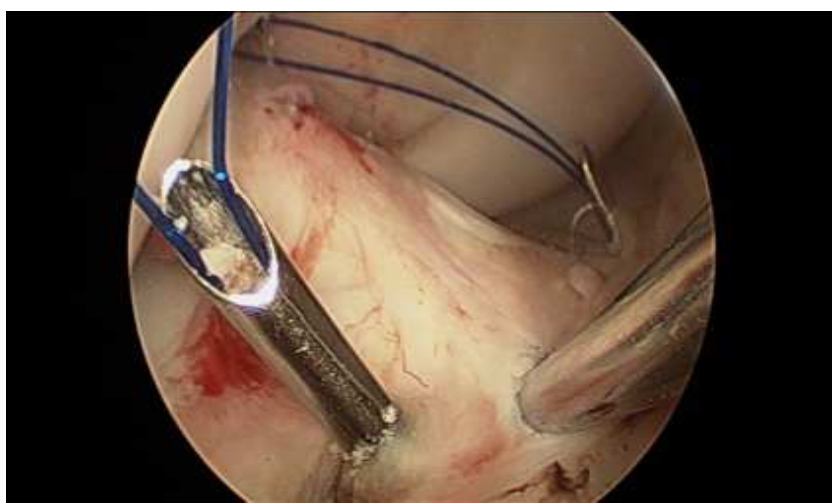

Fig 2: PDS suture passed through both LP needles

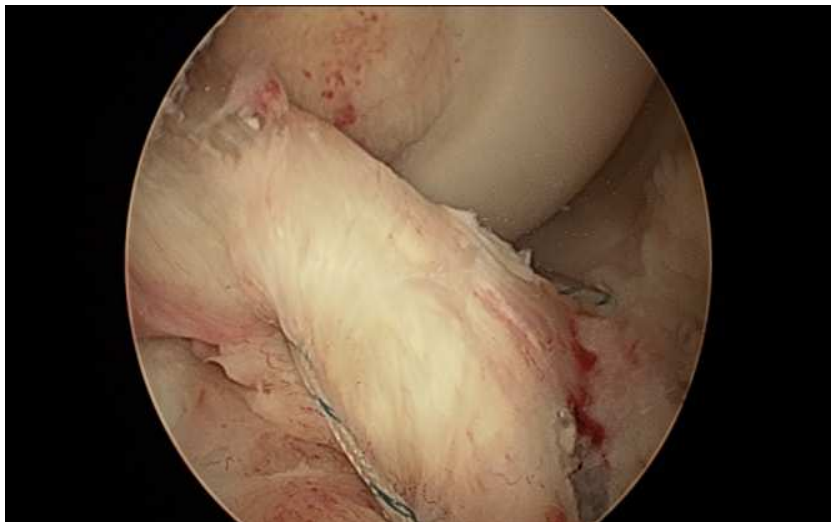

Fig 4: Tibial spine in anatomical position after proper tying of both the ultrabraid sutures.

Rehabilitaion Protocol: Earlier rehabilitation is crucial as it helps in faster recovery and prevention of secondary complications like arthro-fibrosis and loss of extension. Patient's knees were kept in extension with the help of knee immobiliser. Day after surgery range of motion exercises and hamstring stretching exercises in prone position was promoted. Non weight bearing walking was allowed as soon as the pain subsided.

Static Quadriceps exercise was promoted. After three weeks, partial weight bearing was allowed using crutches and full weight bearing was allowed after 6 weeks. Return to Full activity was allowed after 3 months.

\section{Results}

Twenty one patients underwent arthroscopic suture fixation of the comminutedtibial spine fractures. Mean age of the patient was 24.28 years (range 16 to 38 years). All of the patients belonged to the Meyer type IV. The mean duration of follow up was 15.5 months with a minimum of one year follow-up. No patients revealed Quadriceps weakness.

All patients showed negative Lachman test except one having grade 1 positive lachman.No patient showed extension loss.90.4 \% ( $n=19)$ patients had an IKDC grade A while two patients belonged to grade B.All patients returned to their previous activity level. All patients showed radiological bone healing (range six to ten weeks)

\section{Discussion}

Comminutedtibial spine fractures still pose a great challenge to manage being technically demanding procedure. They are more common in children and the adolescent age group [10]. Achieving the biomechanics of the knee joint congruity and ligament integrity are the aims of the treatment. McLennan [11] in 1982, first advocated the advantages of arthroscopic treatment for tibial eminence fractures.

Arthroscopic fixation of the tibial spine fracture is the gold standard technique [3] and the fixation is essential so as to avoid non-union or mal-union leading to residual difficulties of flexion deformity and instability. There are two techniques described for the suture fixation. One is the ACL based (ligament suture 
methods) [12] and the other is avulsed bone fragment based [13]. As in our studies of comminutedtibial spine fractures ACL based suture fixation is advocated as it is very difficult to pass the suture through the comminuted bone fragments. Andalso drilling holes in the comminuted bone fragments can further lead to comminution.

Ligament suture method advocates the use of penetrators or suture passers for passing through the ACL but in our studies we used the LP needle which being inexpensive, also decreases the chances of ACL damage as they are narrow bored and the ACL bites can be repeated.

Screw fixation of the tibial spine fractures has got the dis-advantage of the physeal injury in the children in addition to the second surgery to remove the hardware. Hunter and Willis [14] reported a $44 \%$ re-operation rate in patients treated with cannula tedscrews. Our technique can be safely used in the children without any significant damage by K-wires to the physis.

In the various methods of the fixation of the tibial spine fractures, most significant complication is the residual laxity of the ligament which is mostly encountered due to the inappropriate anterior fixation of the spine. Our method tackles the problem as two loops are used one in the anterior substance of the ACL and other posteriorly thereby decreasing the chances of the residual laxity. Berg et al [15] had reported 2 cases of post operative arthrofibrosis and Montgomery at al [16] reported as high as $53 \%$ of patients had severe difficulty in attaining range of motion post-operatively. In our study, onepatient $(4.76 \%)$ had 1+laxity. No other significant complication was noted.

In our study we used non absorbable (ultrabraid) sutures as they are found to be more stronger and stiffer than absorbable sutures to with stand tensile stresses as supported by the studies of Gerber et al [17].

The above excellent results show that arthroscopic suture fixation of the comminutedtibial spine is promising and reliable technique to achieve the best biomechanics of the knee.

\section{Conclusion}

Arthoscopic suture fixation is a lesser invasive andexcellent technique for the fixation of comminuted fractures of the tibialspine with very good results.
Funding: Nil, Conflict of interest: None initiated. Permission from IRB: Yes

\section{Bibliography}

1. Mann MA, Desy NM, Martineau PA. A new procedure for tibial spine avulsion fracture fixation. Knee Surg Sports Traumatol Arthrosc. 2012 Dec; 20(12):2395-8. doi: 10.1007/s00167-012-1906-9. Epub 2012 Jan 24.

2. Kendall NS, Hsu SY, Chan KM. Fracture of the tibial spine in adults and children. A review of 31 cases. J Bone Joint Surg Br. 1992 Nov;74(6):848-52.

3. Lubowitz JH, Elson WS, Guttmann D. Part II: arthroscopic treatment of tibial plateau fractures: intercondylar eminence avulsion fractures. Arthroscopy. 2005 Jan; 21(1):86-92.

4. Meyers MH, McKeever FM. Fracture of the intercondylar eminence of the tibia. J Bone Joint Surg Am. 1970 Dec;52(8):1677-84.

5. Zaricznyj B. Avulsion fractures of the tibialeminence : treatment by open reduction and pinning. Arthrosc Tech. 2014 Dec; 3(6): e683-e687. Published online 2014 Nov 24. doi: 10.1016/j. eats. 2014.08.008

6. Rockwood CA, Wilkins KE, Beaty JH. Fractures in children. Philadelphia : Lippincott-Raven 1996 : 12961298.

7. Kocher MS, Micheli LJ, Gerbino P, Hresko MT. Tibial eminence fractures in children: prevalence of meniscal entrapment. Am J Sports Med. 2003 May-Jun; 31(3):404-7.

8. Senekovic V, Veselko M. Anterograde arthroscopic fixation of avulsion fractures of the tibial eminence with a cannulated screw: five-year results. Arthroscopy. 2003 Jan;19(1):54-61.

9. Kocher MS, Foreman ES, Micheli LJ. Laxity and functional outcome after arthroscopic reduction and internal fixation of displaced tibial spine fractures in children. Arthroscopy $2003 ; 19$ : 1085-1090.

10. Coyle C, Jagernauth S, Ramachandran M. Tibial eminence fractures in the paediatric population: a systematic review. J Child Orthop. 2014 Mar; 8(2):14959. doi: 10.1007/s11832-014-0571-6. Epub 2014 Mar 2. 
11. McLennan JG. The role of arthroscopic surgery in the treatment of fractures of the intercondylar eminence of the tibia. J Bone Joint Surg Br. 1982;64 (4):477-80.

12. Matthews DE, Geissler WB. Arthroscopic suture fixation of displaced tibial eminence fractures. Arthroscopy. 1994 Aug;10(4):418-23.

13. Medler RG, Jansson KA. Arthroscopic treatment of fractures of the tibial spine. Arthroscopy. 1994 Jun; 10 (3):292-5.

14. Hunter RE, Willis JA. Arthroscopic fixation of avulsion fractures of the tibial eminence: technique and outcome. Arthroscopy. 2004 Feb;20(2):113-21.
15. Berg EE. Comminuted tibial eminence anterior cruciate ligament avulsion fractures: failure of arthroscopic treatment. Arthroscopy. 1993;9(4):446-50.

16. Montgomery KD, Cavanaugh J, Cohen S, Wickiewicz TL, Warren RF, Blevens F. Motion complications after arthroscopic repair of anterior cruciate ligament avulsion fractures in the adult. Arthroscopy Volume 18, Issue 2, February 2002, Pages 171-176.

17. Gerber C, Schneeberger AG, Beck M, Schlegel U. Mechanical strength of repairs of the rotator cuff. J Bone Joint Surg Br. 1994 May;76(3):371-80.

\section{How to cite this article?}

Kumar S, Kushwaha S.S, Singh S, Kumar D, Sharma V. Arthroscopic suture fixation of comminuted tibial spine fractures-a prospective study. Int J Med Res Rev 2016;4 (7):1097-1101.doi: 10.17511/ijmrr.2016.i07.04. 